\title{
Thermal and Fluid Dynamic Analysis of Compact Fin-and-Tube Heat Exchangers for Automotive Applications
}

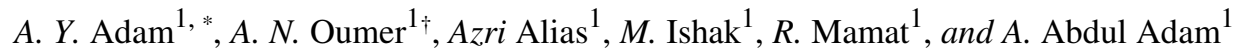 \\ ${ }^{1}$ Faculty of Mechanical Engineering, Universiti Malaysia Pahang, 26600 UMP, Pekan, Pahang, \\ Malaysia.
}

\begin{abstract}
Flat tubes heat exchangers are commonly used in many industrial applications as a consequence of the distinctive geometrical characteristics of the flat tube compared with round tube. This paper aims to investigate the flow and heat transfer characteristics of laminar cross-flow forced convection in compact fin-and-flat tube heat exchangers. The experiment was performed to explore the influence of the tube inclination angle on the thermal hydraulic performance of the flat tube heat exchanger. Four flat tubes arranged in two aligned rows having the same longitudinal and transverse pitches have been examined in the range of Reynolds number between 1768.27 and 2259.46. A constant heat flux of $4169.63 \mathrm{~W} / \mathrm{m}^{2}$ was applied at the inner surface of each flat tube. On the other hand, the numerical simulation is solved by ANSYS FLUENT for a two dimensional model with unstructured mesh and the results are compared against the experimental results. The numerical simulation results indicate that the average Nusselt number increased by $78.24 \%$ for Reynolds number 1768.27. Besides that, for Reynolds number 1964.75 and 2259.46 the Nusselt numbers were increased by $75.89 \%$ and $54.49 \%$, respectively, compared to experimental results. Moreover, the pressure drop is increased $25 \%$ and $83.38 \%$ for both experimental and numerical simulation with respect to three Reynolds number. It was found that, the tube with $30^{\circ}$ degree provides the higher heat transfer with Reynolds number 2259.46. This study could assist engineers in decisions regarding the application of compact fin-and-tube heat exchangers in the automotive field.
\end{abstract}

\section{Introduction}

Currently, fin-and-tube heat exchangers are employed in a wide variety of engineering applications such as modern heat exchangers, automotive radiators, automotive air conditioning evaporators and condensers [1] .In automotive industries, there is a strong necessity of lighter heat exchangers due to the fact that there is direct relationship between fuel consumption and vehicle weight. Moreover, the aerodynamic and aesthetic design restrictions have led to a progressive reduction of the frontal area of the vehicle. The size and performance of compact heat exchangers have significant effect on the end product of automotive components [2,3].

\footnotetext{
* Corresponding author: ahmedyousifam@yahoo.com

† Corresponding author: ahmedyousifam@yahoo.com
} 
For instance, compact heat exchangers involve in engine cooling and air conditions functions of automobiles. Therefore, the design of heat exchangers affects the aerodynamics and performance of the automotive automobile. Thus, an optimized design of the heat exchangers is a key aspect to achieve these trends without losing engine cooling performance. Because of these reasons, an extensive study on the thermal and fluid dynamics of compact heat exchangers is essential.

Considering that an automotive radiator is an important part of the car cooling system, the fin-and-tube heat exchanger is often the subject of research. Many experimental and numerical methods have been proposed to obtain the heat transfer and fluid flow characteristics of fin-and-tube heat exchangers [4-7]. L.H. Tang, M. Zeng and Q.W. Wang [5] have performed an experimental and numerical investigation on air side performance of fin-and-tube heat exchangers with various types of fins (crimped spiral fin, plain fin, slit fin, and fin with delta wing vortex generator (VGs)). Their experimental findings indicated that the heat exchanger with slit fin pattern gave the best heat transfer performance. Moreover, the fins with vortex generator showed the best heat transfer performance over slit fins after optimization. Furthermore, it is also summarized that the vortex generators will bring the overall performance of heat exchanger with VGs better for larger angle of attack, longer length and smaller height. The effects of fin spacing on the performance of heat exchangers were studied by Pongsoi, et al. [8]. They indicated that smaller fin spacing enhances the rate of heat transfer and larger spacing results in an insignificant pressure drop. Besides that, this information is further proved by other research works of Pongsoi et al. [9] that with the different fin spacing effect on the average heat transfer and follow the same trend for the pressure drop with increasing Reynolds number. In addition to the fin spacing, the spacing between the flat tubes has significant effect on the flow and thermal behavior of fin-and-tube heat exchangers [10-12]. For instance, Tahseen, et al. [11] have presented that the increasing in transverse pitch ratio (ST/D) corresponds to increase in Nusselt number and Reynolds number. The results remarked that the heat transfer performance is getting better as the transverse pitch ratio increasing from 3.0 to 6.0 for flat tube in stagger arrangement with longitudinal ratio (SL/D) kept constant.

From the aforementioned literatures, it can be understood that the thermal and fluid dynamics of heat exchangers in automotive applications depend on the shape, type, and size of fins and tubes. In addition, tube spacing and fin spacing can determine the radiator characteristics. Thus, altering these parameters may influence the flow and thermal characteristics of the devise. It can also be noticed from most of the previous research works that circular type of tubes has been used. This is due to the fact that they are the simplest and cheapest. However, the wake region behind the circular tube reduces the heat transfer on downstream fin region [13]. The optimum heat transfer rate can be obtained by varying the geometrical parameters of the tubes. However, the effect of flat tube inclination angle from the incoming air in automotive application has not been studied yet. Hence, the objective of this work is to examine the effect of flat tube inclination angle on the flow and thermal performance of compact fin-and-tube heat exchangers over in-line configuration of flat tube banks with laminar forced convection. The experiment will be performed for four flat tubes arranged in two aligned rows with same longitudinal and transverse spacing for three various Reynolds numbers; $\operatorname{Re}=1768.27,1964.75$ and 2259.46. 


\section{Problem description and experimental apparatus}

The experimental setup used consists of a suction fan, frequency inverter, test section, thermistors, electrical heater, digital manometer, anemometer and straightener. A two row by two column fin-and-tube heat exchanger placed inside a laboratory scale wind tunnel apparatus is used to investigate the tube inclinations angle effects on the flow and heat transfer behavior. The flat tubes arranged in inline configuration are made from aluminum. The dimension of the flat tube is $11.39 \mathrm{~mm} \times 19.88 \mathrm{~mm}$ with tube thickness of $1 \mathrm{~mm}$ as shown in Fig. 1. The flat tube hydraulic diameter $\mathrm{Dh}_{\mathrm{h}}$ is $15.05 \mathrm{~mm}$ and the length of the tubes is $200 \mathrm{~mm}$. The two dimensional cross sectional view of the heat exchanger is shown schematically in Fig. 2. As depicted in the figure, the length, width, and thickness of the fins are $200 \mathrm{~mm}, 145 \mathrm{~mm}$, and $0.6 \mathrm{~mm}$ respectively. The hot fluid heat flux coming from the car engine was simulated by inserting the electric heaters inside the flat tubes for the experiment. Two rows and two columns of flat tubes arranged in an inline manner were designed and assembled as shown in Fig. 2. The test module will be placed into the horizontal wind tunnel for external flow over the tubes. The Teflon type Polytetrafluoroethylene (PTFE) material was used at the two ends of the flat tubes to minimize the heat loss to the surrounding. The test section was adjusted with four different tube angles $(0,10,20$, and 30$)$ in order to investigate the influence of tube inclination angle.

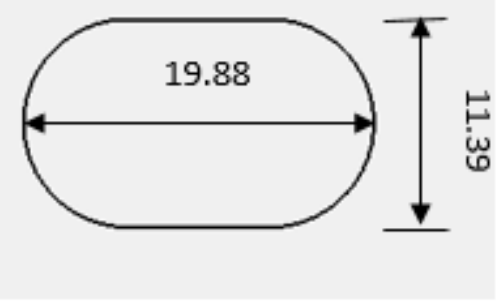

Fig. 1. Dimensions of the flat tube (in $\mathrm{mm}$ ).

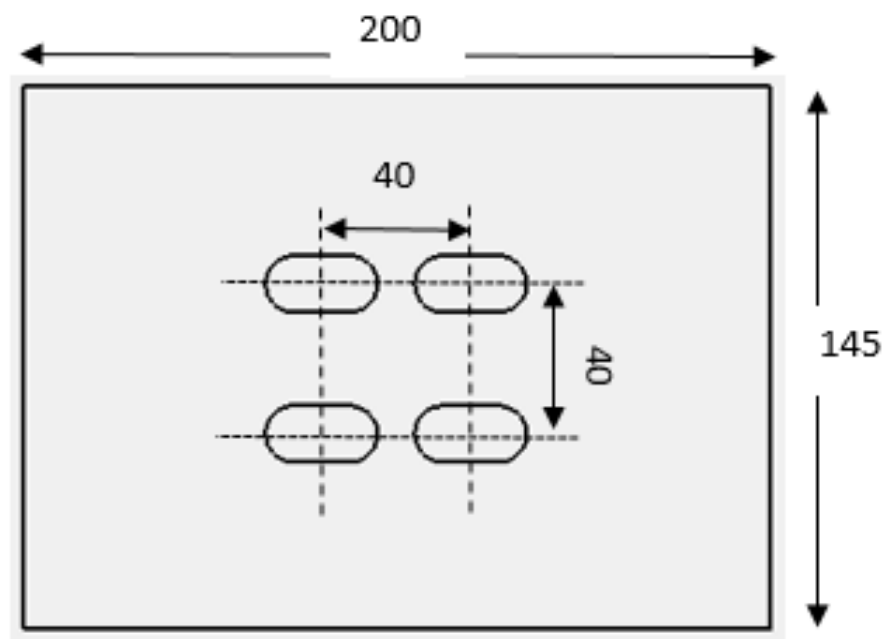

Fig. 2. Cross sectional view of the heat exchanger. 
The AC voltage source of model LOADSTAR 850 was used to heat the flat tubes. This AC source can have maximum supply of $50 \mathrm{~W}$ of power. Four electric heater rods of $8 \mathrm{~mm}$ in diameter and $200 \mathrm{~mm}$ long were inserted into the flat tubes. In addition, the current clam meter and volt meter of models U1191A and TENMA9272, respectively, were used to collect the current and voltage readings. The temperature distributions at the inlet, outlet, on the tube surfaces of the test section, and at the extended region before the test section were collected using thermistors of model EPCOS B57164K01012J NTC. The output resistances of the thermistor during the experiment were converted with the aid of national instrument (NI) and DASYLAB software. Furthermore, the vane type hot wire anemometer was used as the fluid velocity measurement in the wind tunnel when the fan operates to suck the air into the extended region. The model YK-2004AH with accuracy $\pm 0.1 \mathrm{~m} / \mathrm{s}$ was used. On the other hand, the pressure drop was measured using the differential pressure meter of model TESTO 510 with the range of pressure from 0-100kpa. Figure 3 shows the complete experimental setup used in the experiment.

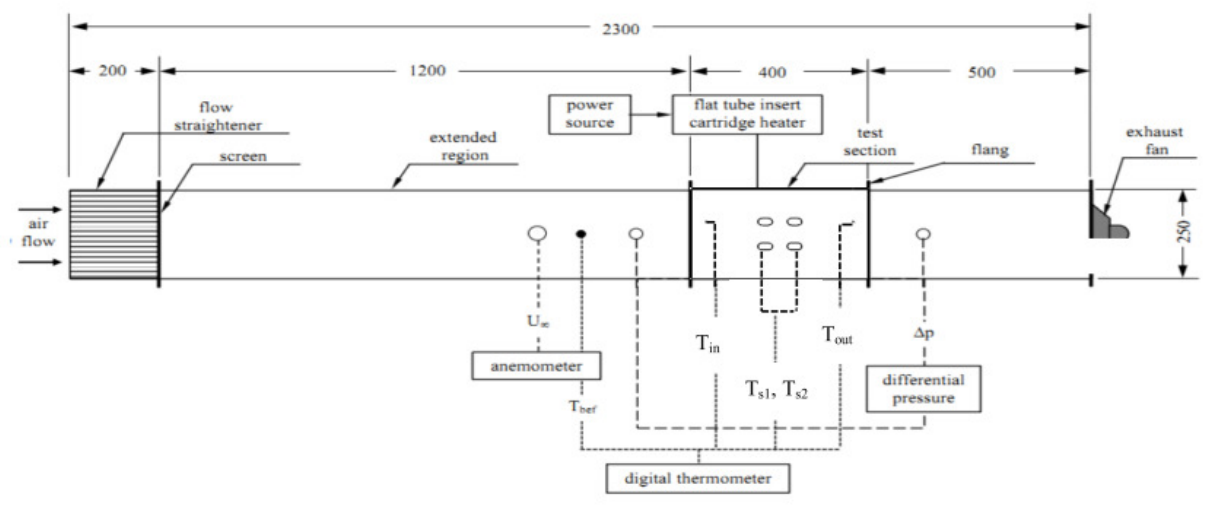

Fig. 3. Schematic of the experimental apparatus.

\section{Numerical modeling}

\subsection{Governing equations}

Modeling of temperature distribution along the fins necessitates consideration of coupled heat transfer between heated tube surfaces, fins and air behavior. The governing equations for the steady state convection for the air flow follow the conservations of mass, momentum and energy, and can be written as follows:

$$
\begin{aligned}
& \nabla \cdot V=0 \\
& (\nabla \cdot V) \cdot V=-1 / \rho(\nabla P)+\left(\mu \nabla^{2} V\right)+\beta\left(T-T_{0}\right) g \\
& V . \nabla T=\alpha \nabla^{2} T
\end{aligned}
$$

where $V$ denotes the velocity vector, $\rho$ the density, $P$ the pressure, $\mu$ the kinematic viscosity, $T$ the temperature and $\alpha$ the thermal diffusivity. 


\subsection{Boundary Conditions}

As discussed earlier, conjugated heat transfer between the flat tube surfaces, the fins and the air flow restricts the problem to only inlet, outlet, and wall boundary conditions. To allow fully developed flow over the tube banks, the selected geometry is extended by some amount as shown in Fig. 4. As depicted from the figure, the air inters from the left side of the heat exchanger at constant velocity and temperature. Also, pressure outlet is imposed as outlet boundary condition, and constant heat flux is applied on the tube surfaces. The boundary conditions applied for the simulations are presented in Table 1. For the simulations, the commercial CFD software called ANSYS FLUENT was used where SIMPLE algorithm was applied to couple the pressure and velocity fields, and power law scheme was imposed to discretize the momentum and energy equations. Boundary layer mesh has been used to detect the velocity and temperature gradients close to the surfaces.

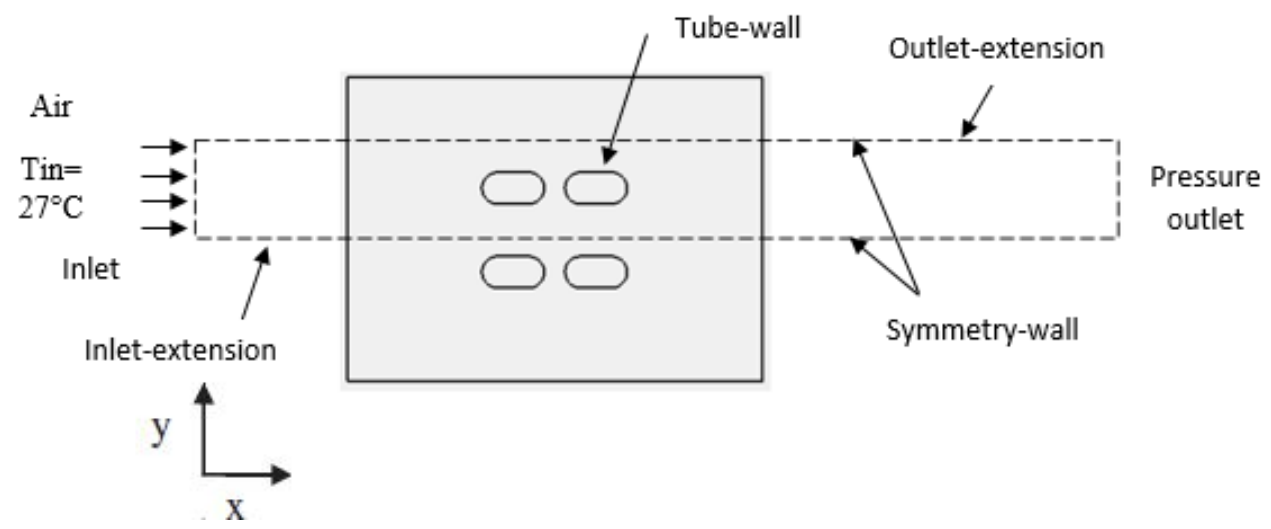

Fig. 4. Schematic and boundary conditions of the heat exchanger model studied.

Table 1. Boundary conditions used in the simulation.

\begin{tabular}{|c|c|c|c|c|c|}
\hline & \multirow[b]{2}{*}{$\operatorname{Re}$} & \multirow{2}{*}{$\begin{array}{l}\text { Surface } \\
\text { flux, } \\
\text { W/m2 }\end{array}$} & \multicolumn{2}{|c|}{$\begin{array}{c}\text { Inlet } \\
\text { parameters }\end{array}$} & \multirow[t]{2}{*}{$\begin{array}{c}\text { Outlet } \\
\text { parameters }\end{array}$} \\
\hline & & & $\begin{array}{l}\mathrm{V}, \\
\mathrm{m} / \mathrm{s}\end{array}$ & $\mathrm{T},{ }^{\circ} \mathrm{C}$ & \\
\hline $\begin{array}{c}\text { Case } \\
1\end{array}$ & 1768.27 & \multirow{3}{*}{4169.63} & 1.8 & \multirow{3}{*}{27} & \multirow{3}{*}{$\begin{array}{c}\text { Zero gauge } \\
\text { pressure }\end{array}$} \\
\hline $\begin{array}{c}\text { Case } \\
2\end{array}$ & 1964.75 & & 2.8 & & \\
\hline $\begin{array}{c}\text { Case } \\
3\end{array}$ & 2259.46 & & 3.8 & & \\
\hline
\end{tabular}

Once the experimental and simulation temperature distributions across the heat exchanger are obtained, the heat transfer rate, the Nusselt number, pressure drop and friction factor are calculated based on the following equations [14].

$$
\begin{aligned}
& Q \operatorname{con} v=h A(T s-T \infty) \\
& h=q /(T s-T \infty)
\end{aligned}
$$




$$
\begin{aligned}
& N u=\left(h D_{h} / k\right) \\
& \nabla P=P_{\text {in }}-P_{\text {out }}
\end{aligned}
$$

where $A$ is the surface area of that convection, $h$ is the convection heat transfer coefficient in $\mathrm{W} / \mathrm{m}^{2} . \mathrm{K}, T s$ is the surface temperature, $T_{\infty}$ is the temperature of the fluid sufficiently far from the surface, $D_{h}$ is the hydraulic diameter, $P_{\text {in }}$ is the inlet pressure and $P_{\text {out }}$ is the outlet pressure.

\section{Results and Discussions}

Before the simulation model was performed to predict the Nusselt number and pressure drop inside the heat exchanger, the model was validated by comparing the results obtained with the experimental data.

\subsection{Temperature Distribution}

Figs. 5 and 6 compare the numerical results and experimental data for the temperature distribution obtained at three different locations in the heat exchanger for the cases of 20 o and 30o tube inclination angels respectively. As expected, the air side temperature increased from the inlet towards the other side of the heat exchanger. According to the figures, acceptable agreement was found between the numerical and experimental data. The maximum percentage errors between the simulation results and experimental data for Figs. 5 and 6 are $7.6 \%$, and $9.6 \%$, respectively. Both the experimental and simulation results indicate that the temperatures decrease as the Reynolds number increase. Moreover, both figures confirm that the temperature changes along the heat exchanger is moderate and definition of Raleigh number based on the average surface temperature will not make a considerable deviation. In additional, the increase in velocity and inclination angles able to dissipate the heat generated faster.

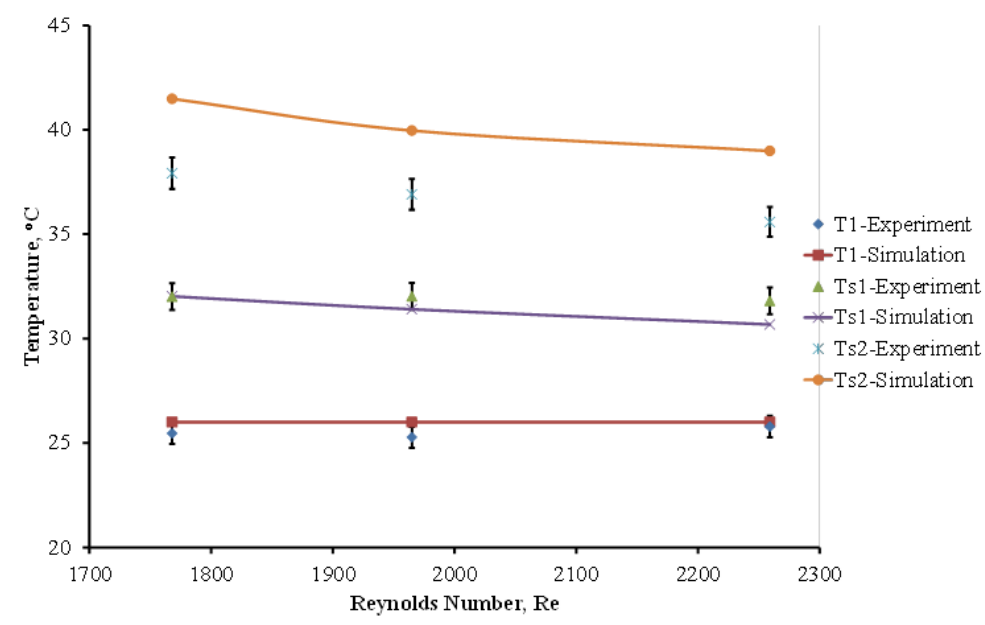

Fig. 5. Comparison of experimental and simulation temperature distribution results across the heat exchanger for 20 degree tube inclination angle. 


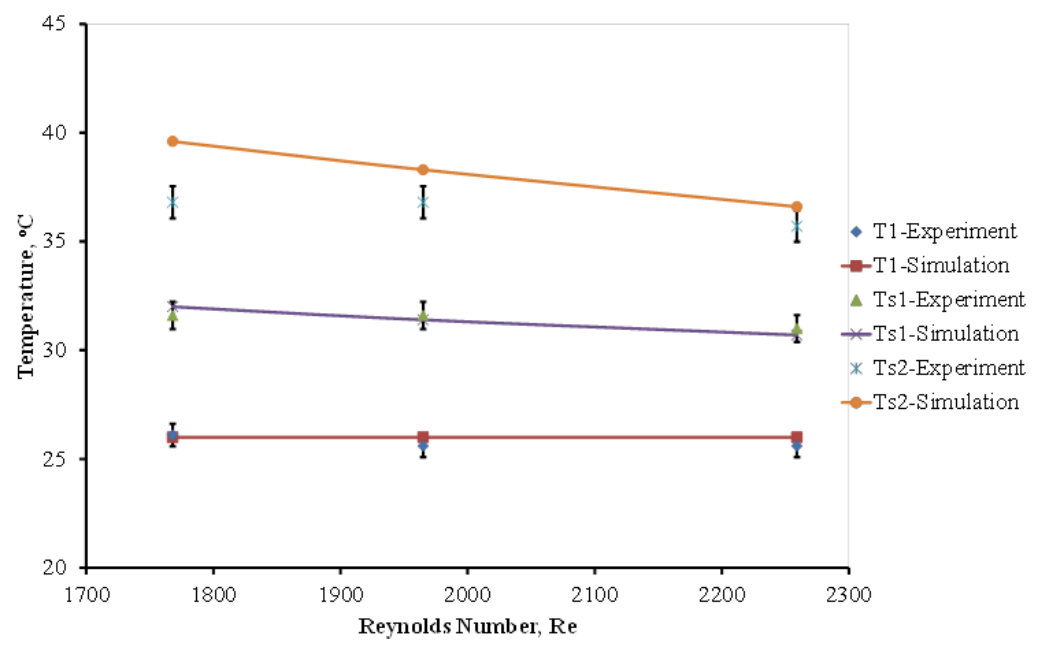

Fig. 6. Comparison of experimental and simulation temperature distribution results across the heat exchanger for 30 degree tube inclination angle.

\subsection{Nusselt number}

Variations of the simulated average Nusselt number $(\mathrm{Nu})$ with the tube inclination angle for three different Reynolds number are shown in Fig. 7. It can be clearly seen from the figure that the Nusselt number at each Reynolds number increased with the increase of inclination angle. Moreover, further increase of the Reynolds numbers may contribute to further improvement in heat transfer. The percentage of Nusselt number increment from Reynolds number 1768.27 to 1964.75 is in the range of $5.04 \%$ to $13.15 \%$. While, the Nusselt number increased from Reynolds number 1964.75 to 2259.46 by $8.19 \%$ to $23.17 \%$. In general, it was observed from the figure that the increase of inclination angle and Reynolds number able to improve the heat transfer. It can be seen from the Nusselt number results that, the heat transfer from the automobile radiator depends on the flat tube inclination angles and the maximum value can be reached at $30^{\circ}$.

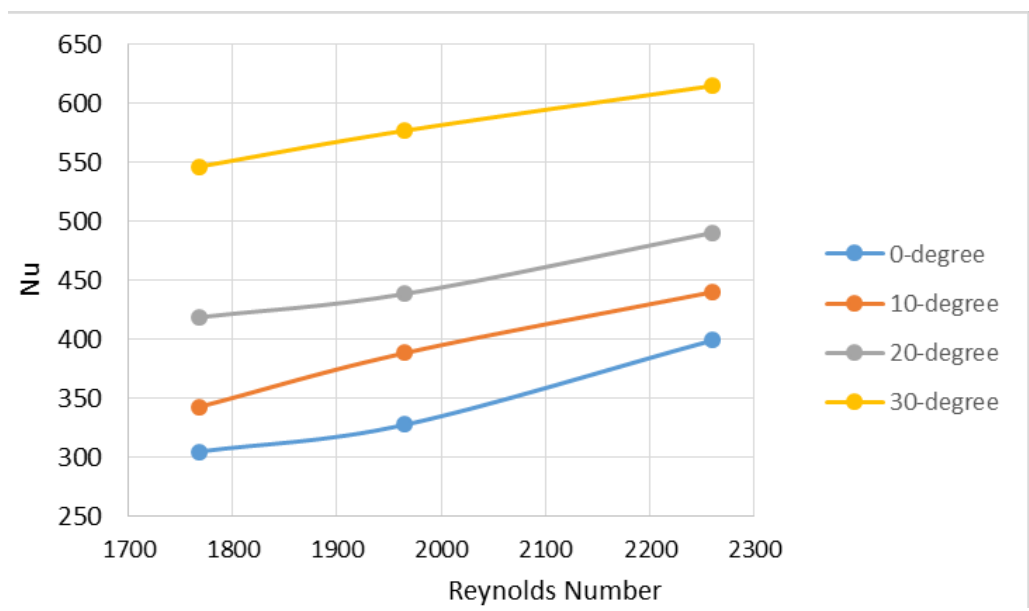

Fig. 7. Variation of simulated Nusselt number against Reynolds numbers with three different inclination angle. 


\subsection{Pressure Drop}

Variation of simulated pressure drop against Reynolds number for $0^{\circ}$ tube inclination angle is depicted in Fig. 8. As expected, the pressure drop increases with the increase of air velocity. It is predicted that increment of the tubes inclination angle would result in an increase in pressure drop. This is due to the fact that restricting a flow path at tube inclination angles greater than $0^{\circ}$ results in turbulence enhancement which in turn results in a high pressure drop penalty. As a consequence, flat tube with the highest tube inclination angle would provide the highest pressure drop. While, the $0^{\circ}$ angles of attack provide the minimum drop in the pressure but associated with low thermal performance as well.

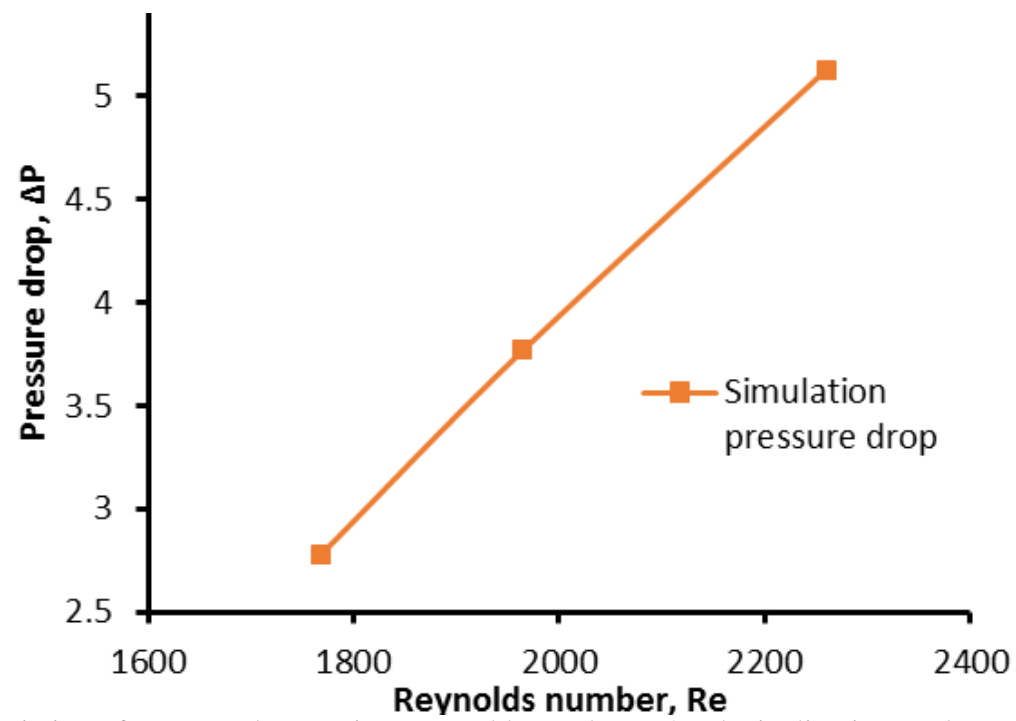

Fig. 8. Variation of pressure drop against Reynolds number at $0^{\circ}$ tube inclination angle.

\subsection{Regression Equation}

The $\mathrm{Nu}$ regression equation for the two by two rows of tube banks was obtained using Minitab 14 software. The obtained regression equation is a second order equation and is valid for inlet air velocity ranges of $1.8-3.8 \mathrm{~m} / \mathrm{s}$ and tube inclination angle range of $0_{\mathrm{o}}-30_{\mathrm{o}}$. The obtained second order equation shown in Eq. (8) expresses the Nusselt number:

$$
N u=209.823+4.23 \alpha+55.43 \mathrm{~V}-1.1965 \alpha \mathrm{V}
$$

Where $V$ is the inlet air velocity in $\mathrm{m} / \mathrm{s}$ and $\alpha$ is the tube indication angle in degree.

Fig. 9 shows comparison between the average Nusselt number obtained from the experiment (Nu-Exp) and the $\mathrm{Nu}$ from the regression equation (Nu-Equation). As can be seen from the figure, the developed equation could provide $\mathrm{Nu}$ results close to the experimental data. The maximum percentage error when using the regression equation is around $7 \%$. Moreover, Eq. (8) indicates that the heat transfer is maximum when using combination of high flow velocity and big tube inclination angle. On the other hand, minimum heat transfer will be resulted when very low flow velocity and small tube inclination angles are considered. 


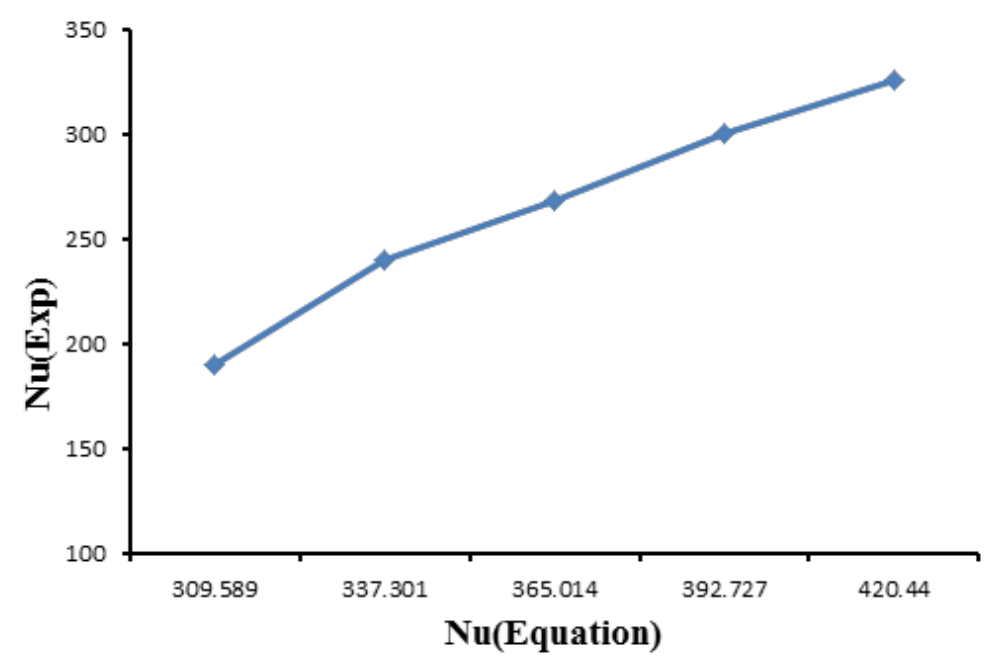

Fig. 9. Comparison between the Nu numbers obtained from the experiment and the regression equation.

\section{Conclusion}

In this study, the air flow and temperature fields were examined for a fin-and-tube automotive heat exchanger. For this, the experimental and numerical simulation results for heat transfer and flow behavior of compact fin-and-tube heat exchangers at various tube inclination angles at three different Reynolds number were analyzed. The tube material was kept fixed as well as the heat exchanger fin and tube pitches. Overall, the experimental and numerical simulation temperature results showed close agreement. The trend of Nusselt number results linearly increased as the tube inclination angle increased. However, the heat transfer improvement due to increase of inclination angle was accompanied by high pressure drop penalty. In generally, the applied numerical studies enable to show the effect of tube inclination angle on the performance of the automotive heat exchanger and can be used as an alternative activity for more costly experimental studies.

The authors would like to be obliged to University Malaysia Pahang for providing the necessary facilities and financial assistance for the study under project number RDU150375. Moreover, the authors would like thank L. Chon for contributing to this work by performing experiments.

\section{References}

1. A. A. Bhuiyan and A. K. M. S. Islam, Int. J. Heat Mass Tran., 101, pp. 38-59, (2016)

2. C. D. Pérez-Segarra, C. Oliet, and A. Oliva, Heat Transfer Eng., 29, pp. 484-494, (2008)

3. C. Oliet, C. D. Pérez-Segarra, and A. Oliva, Heat Transfer Eng., 29, pp. 495-502 (2008)

4. H.-T. Chen, Y.-S. Lin, P.-C. Chen, and J.-R. Chang, Int. J. Heat Mass Tran., 100, pp. 320-331, (2016) 
5. L. H. Tang, M. Zeng, and Q. W. Wang, Exp. Therm. Fluid Sci., 33, pp. 818-827 (2009)

6. R. Sajedi, M. Taghilou, and M. Jafari, Appl. Therm. Eng., 83, pp. 139-146, (2015)

7. Y. Q. Kong, L. J. Yang, X. Z. Du, and Y. P. Yang, Int. J. Heat Mass Tran., 99, pp. 357-371, (2016)

8. P. Pongsoi, S. Pikulkajorn, and S. Wongwises, Int. J. Heat Mass Tran., 55, pp. 65556566, (2012)

9. P. Pongsoi, P. Promoppatum, S. Pikulkajorn, and S. Wongwises, Int. J. Heat Mass Tran., 59, pp. 75-82, (2013)

10. W. Khan, J. Culham, and M. Yovanovich, Int. J. Heat Mass Tran., 49, pp. 4831-4838, (2006)

11. T. A. Tahseen, M. Ishak, and M. Rahman, "Analysis of laminar forced convection of air for crossflow over two staggered flat tubes," Int. J. Auto. Mech. Eng., 6, pp. 753-765, (2012)

12. T. A. Tahseen and R. MM., Int. J. Auto. Mech. Eng., 9, pp. 1487-1500, (2014)

13. P. Wais, "Fin-tube heat exchanger performance for different louver angles," Zeszyty Naukowe Politechniki Rzeszowskiej. Mechanika,( 2014)

14. N. T. Rao, A. N. Oumer, and U. K. Jamaludin, J. Supercrit. Fluid., 116. pp. 132-147, (2016) 